\title{
How to Build Multimedia and Multichannel Corporate Brochures
}

\author{
Nicoletta Di Blas \\ HOC-LAB Politecnico di Milano \\ nicoletta.diblas@polimi.it
}

\author{
Paolo Paolini \\ HOC-LAB Politecnico di Milano \\ paolo.paolini@polimi.it
}

\author{
Elisa Rubegni \\ University of Lugano \\ elisa.rubegni@usi.ch
}

\begin{abstract}
This paper is about an innovative approach for building, at low cost and in quick time, effective multimedia brochures that can be delivered through multiple channels: as web applications, as downloadable applications (for example, for iPod or smart-phone), as off-line applications (for example, CD-ROM, USB), as informationpoints, as standard (interactive) telephone applications, and so forth. An approach, a methodology, and a toolkit (1001stories) allow all of the above, as demonstrated by several case studies, some of which are presented in this paper.
\end{abstract}

\section{Introduction}

Technology has brought about significant changes in the way the corporate world communicates. As at the beginning of the Internet era a website was a must-have, now the need for a more innovative form of digital communication is rapidly spreading. In this paper we present an approach, an innovative methodology and a toolkit (1001stories), that enable users to generate multimedia corporate brochures in a quick, cost-effective way, with a number of highly desirable features:

- a very innovative multimedia and multi-channel form of communication is created, at the same time (that is, delivered over multiple channels: the web, smartphone/iPhone, iPod, CD-ROM, standard cellular phones, and so forth );

- a narrative communication style that allows conveying even difficult/technical content in an engaging way is exploited;

- the methodology and the tool are very easy to learn (no particular technical knowledge is required); therefore, they potentially can be used inside an organization to update, amend, and adjust the brochure over time by a staff of nontechnical authors and without significant effort [1];

- the resulting communication is easily customizable, in the sense that given a bulk of basic content, new stakeholders can add/subtract/adjust content in order to create their own personalized brochure (we call this process the "communication supply chain").

The paper is organized as follows: first of all, we introduce the 1001 stories toolkit and its production workflow. We then present three case-studies of corporate brochures produced with 1001stories: Mechatronic (a Swiss company producing spindles); EPSON Italy (to advertise one of their printers); and the Italian Ministry for Agriculture, Food, and Forests about organic chicken. We then discuss the above four strong points of the toolkit, finally concluding with an overview on the pros and cons of the approach and future research directions.

\section{The 1001stories toolkit}

In November 2005, HOC-LAB was asked by the Pinacoteca Ambrosiana di Milano to produce digital support for an exhibition about the Renaissance painter Bramantino that was about to take place in December. Being under pressure for the short time constraint, we had to devise an approach that could guarantee, at the same time, a high quality result, simplicity of the authoring, and quick delivery. The result was a successful website that could also be delivered as a CD-ROM for the exhibition's visitors.

After the successful completion and delivery of this work, we decided we could expand and engineer the approach keeping in mind a few strong points: quick delivery, effective workflow (especially for content production), engaging narrative style, avoidance of too expensive multimedia (such as shooting new videos rather than using existing footage), and overall low-cost. In short, from that experience, the 1001stories toolkit was born, composed of a design schema, a dedicated engine (consisting of an authoring environment and a generator), a precise production workflow and a communication model that steers the author's choices in preparing the content. In the rest of this section we will describe, in some detail, these features. 


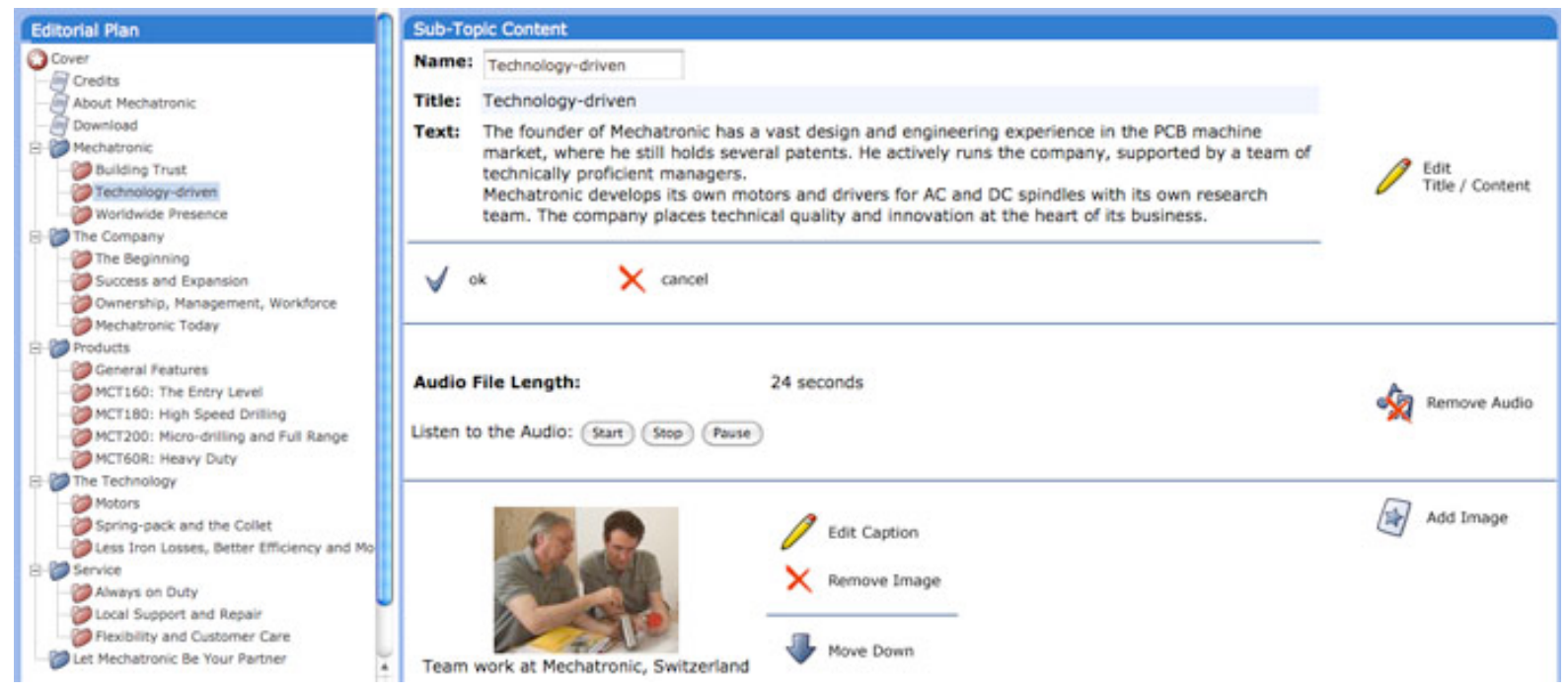

Figure 1. Data entry using the 1001stories engine. On the left, the tree-structure of the content (topics and subtopics); on the right, the content editing functions.

\subsection{The design schema}

The whole content is organized into chapters. Each chapter is composed of a topic and a set of sub-topics (see Figure 1). As regards navigation, the user can get either the set of main topics that, being conceived as executive summaries of their sub-topics, provide an overview of the whole content (this type of use we call "automatic short navigation"), or the complete set of contents ("automatic long navigation"). She is of course free, as a third option, to select each item separately ("manual navigation"). Additional options are the loop navigation, either the short or the long version, which is quite useful during exhibitions or fairs. A number of usage models are therefore available for the user to choose. Each content item (that is, the topics and the sub-topics) is composed by an audio commentary (the transcript of which is available on demand) and visual communication (flash animation, and/or slideshow, and/or videos).

\subsection{The engine}

In essence, the functionalities offered by 1001 Stories are three: Data Entry, Preview and Generator. The Data Entry is a control panel enabling the user to edit the editorial plan, to enter content (that is, title, text, images with captions, and audio file), and to perform all needed edits (Figure 1). The Preview allows the user to visualize at any moment of the process the preliminary resulting story (as it will appear to the end user) of what has been entered thus far. In this way, the developer can immediately check the quality of the story and improve it. The Generator produces and publishes the final application for the various delivery channels. Figures 2 and 3 show examples of a narrative that was built using our tool, delivered on a stationary on-line (or off-line) channel and on iPhone.
From a technical point of view, 1001Stories is a Web application whose architecture relies on three main conceptual modules that operate independently over the same data source. The front-end module renders a story in a chosen graphics and layout template; this module manages the exploration of the story episodes. The authoring module manages the story's editing and allows the creation of multimedia episodes in an intuitive way; furthermore, it offers administrative functionalities (users management, privileges, projects' management, and so on). The third module handles the stories exporting formats and can generate CD-ROM, RSS feeds, podcasts, and possibly even more in the near future. The implementing technologies are MySQL as DBMS and PHP as scripting language.

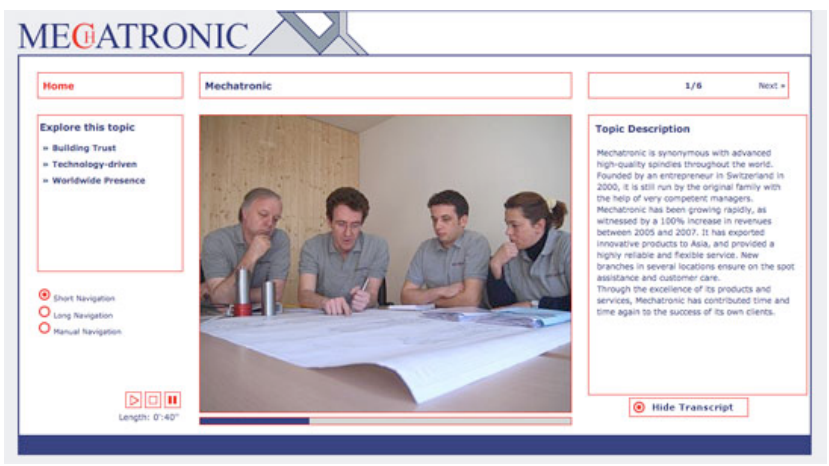

Figure 2. The Mechatronic brochure's stationary version (web). On the right, the audio's transcript is visible. On the left, the list of sub-topics for the current topic (title on top of the image). 


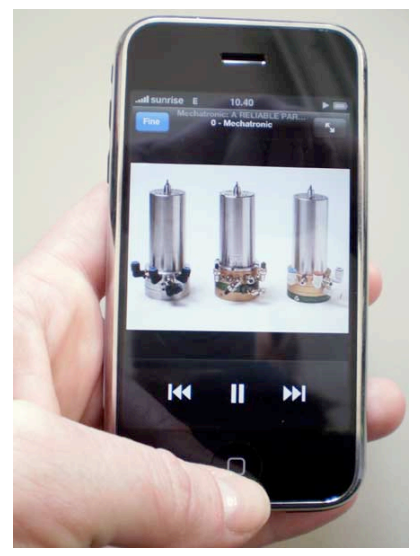

Figure 3 The Mechatronic brochure on mobile device (iPhone).

\subsection{The production workflow}

The production workflow is one of the strong points of the toolkit and is expressed in a short user's manual [3]. It is composed of 10 steps:

1. Definition of the overall communication strategy: subject, target, goal.

2. Definition of the editorial plan: chapters (topics) and their details (subtopics).

3. Definition of the visual communication: even with just the titles of chapters and details, it is possible to start looking for images, videos, animations, and so on.

4. Writing of the texts: the most effective way to gather the content is to interview a subject matter expert and refine the transcript. A 3-hour interview can provide the content for a mid-sized application.

5. In-home recording of the audio: texts are recorded by the best voice on the staff.

6. Creation of the first version of the narrative, putting together visuals, texts, and audios.

7. Quality check: the quality check is performed by using the application in all possible ways (that is, exploiting all kinds of interactions).

8. Revision: texts (and therefore the corresponding audios), captions, and images are changed and amended as required by the quality check's results.

9. Professional speaking: this is a major step. From this moment on, changes to texts cannot be made, unless very necessary, for budget reasons (the professional speaker must be hired again even to change a single word).

10. Creation of the final version. This version can be further adjusted without significant effort as regards images and captions. Changes to audio should be avoided.

The average time for producing a brochure is one month.

\subsection{The communication model}

The applications produced with 1001 stories all correspond to a communication model with the following characteristics:

- The content is split into items (the above mentioned topics and sub-topics). These items are meant to be consumed in a sequence (for example, in the short navigation) but also individually (in the manual navigation), therefore they are, to a certain degree, autonomous. We translate this requirement in the user's manual by asking users to avoid any explicit linguistic reference to other items (such as "as we said before" or even "this" referring to a previously mentioned item). The result is a galaxy of small pieces of content with trails (the chapters) that link them.

- The content items are small. Each content item is composed of audio and some kind of visual communication (slideshow, video, flash animation). The audio is typically no longer than 2 minutes, with 1 minute the ideal length. This makes the consumption more pleasurable: 1-2 minutes for a piece of content is an acceptable time for the user; if she wants to know more, she just has to let the navigation go and relax, but there are plenty of full stops where she can end the experience.

- Audio is the king. The visual communication is there to evoke concepts. There are two reasons for this. First, it makes the production workflow more effective: strict synchronization is time-consuming and expensive. Second, if text and therefore audio is the king, a user can get the message even without looking at the screen. This can be useful in many situations (such as while driving a car, visiting a museum, and so on) - and even necessary, for example in the case of visually-impaired users.

- The narratives are lightweight in terms of content and their goal is to provide an overview of a subject matter and raise curiosity about it. The editorial plan (that is, the organization of the topics and subtopics) is therefore quite loose.

In addition, when producing brochures (narratives with persuasive purposes) we adopt what we call an "argumentation schema." By this we mean that through indepth discussion with the stakeholders we identify some key values the brochure is meant to express. We call this set of key values the argumentation schema, which is fundamental for orienting the creation of content and for making the narrative consistent in all its parts. For example, in the case of Mechatronic, two key values were identified: "Mechatronic is a reliable partner" and "Mechatronic works with Swiss-quality standard." Pictures and texts were carefully chosen to convey these messages (see Figure 2, where an almost familial gathering around a table is shown). 
This model's requirements can be traced back to the Bramantino application: easy production (to allow a staff of non-techy people - our own staff! - to be able to produce the applications), quick delivery (that implies, for example, lightweight content, no strict synchronization between text and images, and so forth), but also communication efficacy (the capacity to raise interest). These requirements and their solutions were generalized in the "instant multimedia" approach [3]. Extensive user studies, conducted around cultural heritage narratives, demonstrate its positive impact $[6,7]$.

\section{Creating multimedia brochures}

From the first narrative (December 2005), more than 40 professional applications have been developed by both HOC-LAB (Department of Electronics and Information, Politecnico di Milano, Italy) and TEC-LAB (Faculty of Communication Sciences, Università della Svizzera italiana, Switzerland). Besides heritage (the original application domain [2,3]), other areas were involved, including corporate communication. In this section, we present three case studies that well exemplify how 1001stories can be used for the production of corporate multimedia brochures.

\subsection{Mechatronic}

The Mechatronic group is a Swiss company specializing in the production of spindles. In year 2007, they asked the TEC-LAB of the Università della Svizzera Italiana of Lugano for an application with a promotional purpose, aimed at suppliers, their current customers (to reinforce the relationship with them) as well as potential new customers.

In order to define the argumentation schema, the best possible arguments, and therefore the most suitable content, the founder and the chief engineer were actively involved from the first stages of the process. They wanted to present the company as young, flexible, and reliable and their products as highly innovative and marked by Swiss quality. Therefore, when preparing the content, both the high level of expertise of the members and the familiarity of the work environment were highlighted (for example, Figures 2 and 3 ).

The brochure is composed of six chapters (Mechatronic, The Company, Products, The Technology, Service, Let Mechatronic Be Your Partner) and it lasts approximately 25 minutes. Given the fact that the company has offices in Asia, the brochure was duplicated in English, Chinese and Korean (www.mechatronic.usi.ch).

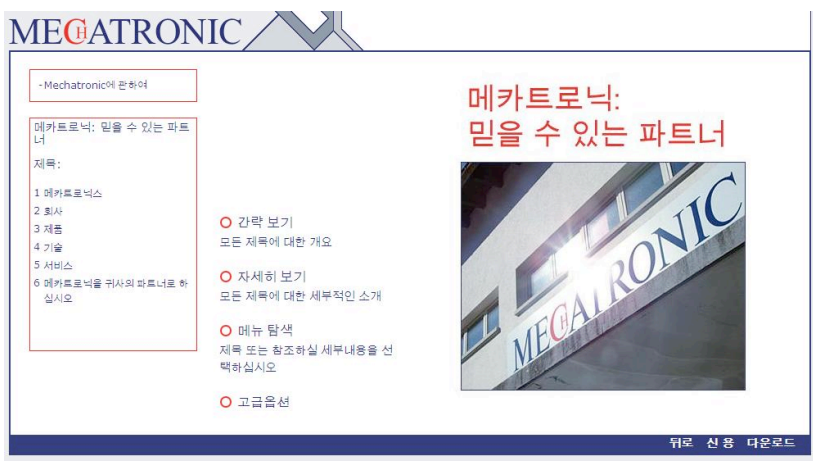

Figure 4. The Chinese version of the MM brochure "Mechatronic: a reliable partner"

\subsection{EPSON's printer}

In January 2009, HOC-LAB was asked by EPSON Italy to produce a brochure about their new EC 01 printer, the first printer without a cartridge. The goal of the brochure was to praise the printer's merits, specified as easy to use, cost-effective, and green. Its target included schools (at any level), companies, and public institutions.

This brochure required a strong argumentation component: for example, our clients were well aware that they needed to persuade their customers that even if the price was higher, the printer, at the end of the day, was still more economic than normal printers.

The overall application lasts approximately $30 \mathrm{~min}$ utes. The main chapters are dedicated to a general presentation (chapter 1: an innovative printer), to the printer's possible targets (chapters 2-4), to the printers environmental friendliness (chapter 5), and in the end to information on how to get it (chapter 6). The images underline the main concepts or suggest possible scenarios of use, including a spaceship! The brochure is available at www.epson.it/Stampanti-e-multifunzione/Stampanti-emultifunzione-inkjet/Epson-EC-01 and also distributed to local retailers of EPSON to be used as marketing tool.

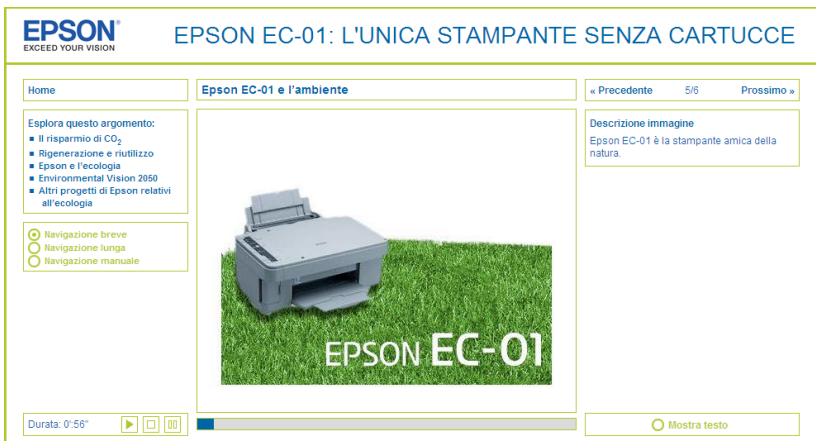

Figure 5. The brochure about the printer without cartridges - EC01, by EPSON - is built upon three main keywords: economic, green, and easy to use. 


\subsection{Italian Ministry for Agriculture}

In spring 2009, HOC-LAB created a narrative about organic chicken in the frame of a national project promoted by the Italian Ministry for Agriculture. Organic food generally speaking looks worse and costs more than other offers on the shelf. Why should a consumer buy it? The project's aim was therefore to build a prototype of effective communication about organic food's quality based on multi-channel technology capable of reaching the consumer in critical situations, like at the supermarket in front of the shelf, at the restaurant, at home, on the train etc.

As case study the organic chicken was chosen. In this example, an expansion of the communication model was devised: the so-called "communication supply-chain". The basic idea is that different pieces of information can be combined when needed just like building blocks. For example: a scientific institution builds the basic building block with general information about the organic chicken. Than a breeder can borrow this bulk of information and add her own pieces of information like what specific races of chickens she breeds, where, etc. (together of course with her own pictures). The breeder's multimedia brochure is therefore created without much effort! Third, a distribution point can borrow some of this already made material, adding new personalized content etc.

Two prototypes were built: one exemplifying the basic building block (the organic chicken in general) and another exemplifying the breeder's brochure (http:/hoc.elet.polimi.it/biopollo/). Both brochures can be found on Youtube (fig. 6) and Flickr.

\section{Why this approach is desirable}

Our experience in creating multimedia brochures as well as other genres of professional applications has led us to conclude that there are four main crucial pros that make this approach desirable.

- Innovativeness. With a non-conspicuous effort (both in terms of budget and man power), a highly innovative form of communication is created. The brochures are at the same time multimedia (therefore pleasurable to consume and rich in terms of variety of content) and multichannel (they are delivered over the web, CD-ROM, smartphone, iPhone, iPod, standard cellular phone-audio only, IVR version - and the main social media: YouTubesee Figure 6-Flickr). Additional features might be Bluetooth advertising (for example, to tell the consumer when she is in front of a store shelf that information about what she is buying is available) and optical labels. In a word, the user is reached anytime, anywhere, with a non intrusive and pleasant form of highly innovative communication.

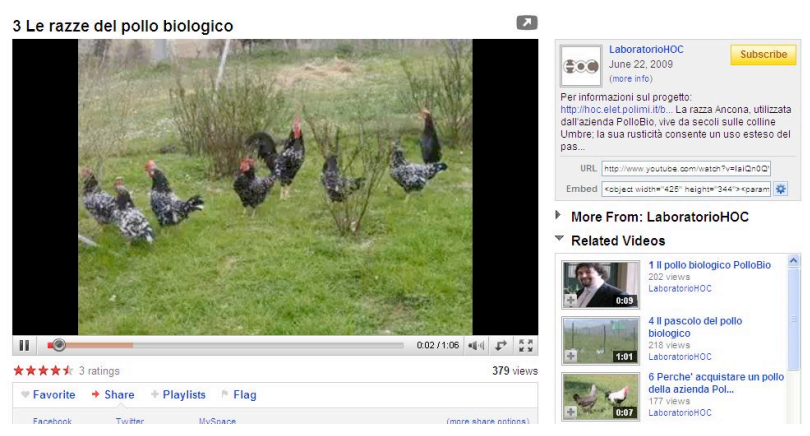

Figure 6. The brochure about organic chicken on YouTube. The efficacy of this communication channel was proved by the fact that although we clearly stated that the video was a prototype by a university lab, still a local butcher called us to actually buy organic chicken from us.

- Understandability. We call our brochures "narratives" in that their style must be easy to understand. One of the big advantages is that even when dealing with difficult content (like for example is the case for cultural heritage or technical issues) we push our experts to be as simple as possible. The way content is gathered, by means of an oral interview, and then refined (according to a set of rules of style, including instructions on the syntax, the length of the texts, the linguistic intra-textual referenceswhat must not be there, and the like) makes the final audio not only easy to grasp but even pleasurable to listen to.

- Ease of use. As we said above, HOC-LAB created an easy-to-use tool to allow HOC's own staff to produce the multimedia narratives. The proof that the 1001 stories engine is really easy to use is that every year, from 2006, hundreds of Italian schools use it to take part in a national competition (www.policultura.it; $[4,5]$ ). They are given a user's manual (12 pages, most of them concerning how to build a narrative and only a couple being about how to use the engine) and there is an help-desk, which basically none uses: they can manage the tool by themselves. The average learning time measured with kids from primary school is approximately 20 minutes.

- Possibility of customization. As explained in the organic chicken case-study, our approach is highly customizable and at low cost, in terms of budget and effort. The communication supply chain means that content (images, videos, texts, audios...) can be added/removed/adjusted at will and also according to budget constraints. Getting back to the organic chicken example: a few hundred Euros are enough for a breeder to add one/two chapters to the general story (Figure 7). 


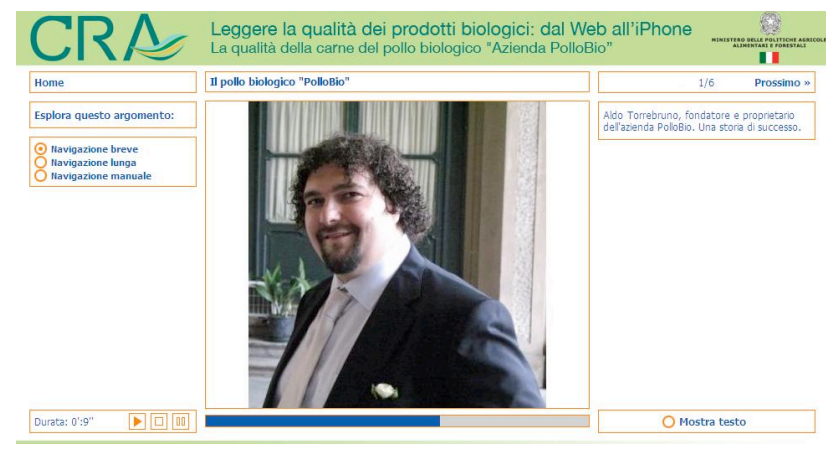

Figure 7. An example of a "communication supplychain": a breeder adds his own information to the general brochure about organic chicken, thus creating his personalized brochure.

\section{Conclusions}

On the basis of our experience, and on the current trend in the field of professional communication, we propose our vision for multimedia corporate communication:

- The communication must be fast, agile, and reactive: focusing, at the proper time, on specific messages and targets is more effective than static, generalist communication.

- Low-cost productions have a big advantage: whatever the budget, it is better to use it in small pieces rather than in a big chunks. Small budget projects can be developed quickly and on time, and revised often.

- The variety of channels is an absolute must. The user should be allowed to use any device or any connection technology, not being confined to a restricted set of choices. The web application should be accompanied by podcast, smart-phone, iPhone, or iPad now, as well as information points, and even standard telephone (voice only).

- The presence in social spaces (for example, Youtube, Flickr, but also blogs and the like) should also be considered as a necessary ingredient.

- The different actors of the same communication supply chain (say, for example, tourism, or culture or organic food) should be able to exchange content, creating high value multimedia brochures at low cost.

Combining all the above is not easy, and that is why we keep on working on our toolkit in a number of directions, all of them aiming at providing a better service to corporate communication professionals.

\section{References}

[1] Bolchini, D., Di Blas, N., Garzotto, F., Paolini, P., Torrebruno, A. Simple, Fast, Cheap: Success Factors for Interactive Multimedia Tools, PsychNology Journal, Volume 5, Number 3, $253-269,2007$
[2] Caporusso, D., Di Blas, N., Franzosi, P., A Family of Solutions for a Small Museum: The Case of the Archaeological Museum in Milan, in Bearman D. \& Trant J. (eds), (2007) Museums and the Web 2007. Selected Papers from an International Conference, Archives \& Museum Informatics, San Francisco, California, U.S.A., 2007

[3] Di Blas, N., Bolchini, D., Paolini, P., Instant Multimedia: A New Challenge for Cultural Heritage. In: Bearman, D. \& Trant, J. (eds.) Museums and the Web 2007. Published at http://www.archimuse.com/mw2007/papers/diBlas/diBlas.html. Accessed 10 February 2010

[4] Di Blas, N., Garzotto, F., Poggi, C., Torrebruno, A., "Instant Multimedia" for Educational Setting: A Success Story. In: $E D$ MEDIA 2008, pp. 538-544. AACE, Chesapeake, VA, 2008

[5] Di Blas, N., Boretti, B., Interactive storytelling in pre-school: a case-study. In: 8th Int. Conf. on Interaction Design and Children, pp. 44-51. ACM, NY, 2009

[6] Di Blas, N., Rubegni, E., Paolini, P., Enigma Helvetia, Promoting an Exhibition through Multiple Channels, Information Access to Cultural Heritage (IACH) workshop, Organised in conjunction with the 12th European Conference On Research And Advanced Technology For Digital Libraries (ECDL 2008), September 18, 2008, Aarhus, Denmark

[7] Franciolli, Rubegni E., Paolini P., Multimedia Communication Issues: Why, What and When. In: Bearman, D. \& Trant, J. (eds.) Museums and the Web 2010. Published at http://www.archimuse.com/mw2010/papers/francioli/francioli.ht ml. Accessed 18 April 2010

\section{About the Authors}

Nicoletta Di Blas is a researcher with the Department of Electronics and Information of Politecnico di Milano. She teaches communication theory for Politecnico di Milano (Como campus) and communication for cultural heritage for the University of Lugano (Switzerland). She is staff member of the HOC-lab of Politecnico di Milano (http://hoc.elet.polimi.it) in projects related to the following areas: (1) multimedia, multichannel applications; (2) serious games design (3D collaborative worlds, digital storytelling tools); (3) impact evaluation (on both fields of application).

Paolo Paolini is full professor at Politecnico di Milano (Italy), adjunct professor at the School of Communication Sciences, and scientific coordinator of the master TEC$\mathrm{CH}$ (Technology Enhanced Communication for Cultural Heritage) at the University of Lugano (USI). He is the scientific coordinator of HOC-lab of Politecnico di Milano (http://hoc.elet.polimi.it), where, since 1998, he has managed several projects related to ICT-based communication for cultural heritage (websites, multimedia, multichannel applications), eLearning (on-line courses, multiuser 3D environments for education) and eTourism. 
Elisa Rubegni is a postdoc researcher at the Università della Svizzera italiana $(\mathrm{CH})$. She holds a Ph.D. in computer science from the University of Florence (IT), and she graduated in communication science at University of Siena (IT) specializing in Human-Computer Interaction.
She teaches usability and interaction design at the Università della Svizzera italiana both at the master's level (TEC-CH-technology-enhanced communication for cultural heritage) and at the bachelor's level (on the Communication Science faculty). 